\title{
Urban morphology in China: origins and progress
}

\author{
Ming Liu, Feng Song* \\ College of Urban and Environmental Sciences. Peking University. Beijing. China \\ *(corresponding author) \\ E-mail: liumingpku1992@163.com,songfeng@urban.pku.edu.cn
}

\begin{abstract}
This paper traces the origins and development of indigenous urban morphological research in China. It also considers the adoption of the theories and methods of the Conzenian School. Urban morphological research in China is carried out in different disciplines: mainly archaeology, geography, and architecture. The earliest significant work was within archaeology, but that has been widely ignored by current urban morphological researchers. As an urban archaeologist whose first degree was in architecture, Zhengzhi Zhao worked on the Studies on the reconstruction of the city plan of Ta-Tu in the Yuan Dynasty in 1957. He uncovered the original city plan of Ta-Tu (now Beijing) in the Yuan Dynasty by applying street pattern analysis. Before the Cultural Revolution, Pingfang Xu recorded and collated the research findings of Zhao, who was by then seriously ill, so that the methods he developed could be continued with the help of other scholars especially archaeologists. His methods of study are still used in studies of urban form in China today. Later, the dissemination of the Conzenian School of thought, aided by two ISUF conferences in China, promoted the development of studies of Chinese urban form. With the help of Jeremy Whitehand, researchers, including the Urban Morphology Research Group of Peking University, applied the theories and methods of the Conzenian School through field work and empirical studies. Taking the opportunity of the 110th anniversaries of the birth of both M.R.G. Conzen and Zhengzhi Zhao, this paper summarizes multidisciplinary urban morphological research in China.
\end{abstract}

Keywords: Urban morphology, disciplinary history, Conzen, China.

\section{Introduction}

Similar to what has been experienced in developed countries in Western Europe, cities have entered a stage of rapid urbanization in China. Although there are significant differences in history, culture and social between China and Europe countries, the urban problems are the same. The rapid changes and disappearance of historical urban landscape, the difficulties in protection work of historic settlements, these issues have arisen in developed countries are now appeared in China. As a result of the specificity of land system and planning policy, revolutionary changes make the situation tougher in landscape protection. Urban morphology, as an important theoretical basis for the protection of historical landscapes, has a wide range of applicability in Chinese cities. However, compared with developed countries, the lag of urban development in China leads to hysteresis of the public policy and theoretical discussion. The research of urban morphology began relatively late, but there are still a number of prospective researchers focused on and explored the knowledge of urban form early, and achieved very important results.

Regrettably, some important researches have been neglected for decades because of the negative academic environment in China in 1970s and tighter bound of disciplines in the second half of the twentieth century. Now we are gradually aware that within the field of urban morphology this has maintained, if not 
exacerbated, a more fragmented knowledge than that contributed by our predecessors of the first half of twentieth century. Therefore, interdisciplinary vision is important and necessary in the study of urban morphology. With the perspective of multidisciplinary, retrospecting the urban form researches in China and analysing its developing process and impetuses not only to evoke the attention to researches done by previous generations and promote their continuation, but also to make new prospects of urban morphology research in China.

\section{Traditional studies on Chinese urban form}

The origin of study on Chinese urban form

One of the earliest Chinese scholars who began the study on urban form was Zhao Zhengzhi, a scholar with architectural background. Although he did not use the term "urban morphology" in his study, his case study on the capital plan, consideration on historical factors of Yuan Dynasty Beijing City and its relation with modern Beijing agree with some methods and contents of urban morphology studies in Conzenian school. Unfortunately, after ZHAO Zhengzhi first published his report of Case Study on Yuan Dynasty Beijing City in 1957, he was diagnosed with cancer in 1960. Later Mr. XU Pingfang recorded and reorganized his related study results from his dictation, forming the Restoration study on urban plan of Yuan Dynasty Beijing City. Influenced by the Cultural Revolution after ZHAO's death, it was not until 1979 that his study was published on the Corpus of the Science and Technology History. the most remarkable achievement in ZHAO Zhengzhi's case study was the restoration of the planning methods by inferring the street pattern as well as quantitative analysis of plot scales.

ZHAO believes that most of the streets in Yuan Dynasty Beijing City, except the main streets of south-north directions, are parallel Hutong of east-west direction. The streets and Hutong in the north of Chang'an Avenue of the inner city of Beijing today, was followed by the plan of Yuan Dynasty Beijing City.
A number of parallel planned street pattern with the same spacing (50 bu, 50 steps) can still be found in the modern city of Beijing, which are the remains of street pattern of Yuan Dynasty Beijing City. In the analysis of street pattern remains, Zhao also noted that with the passage of time, different plots appeared to undergo regeneration of different intensities, tremendous changes in function, scale and form have occurred in many enclosed plots, which in fact the translation and replacement process were pointed out, and the result of plot segmentation and reuse were noted. But as a palimpsest, the city is not completely erase traces of history, part of the center of buildings and courtyard traces have been retained, according to which ZHAO restored the street distribution and some important building sites such as imperial city, palaces and temples.

In the restoration of the urban plan of Yuan Dynasty Beijing City, ZHAO infer the location and spatial characteristics of the moat by existing street pattern. He noted that intersections of two lanes, Maxian Hutong and Anfu Hutong, face the Wengcheng (small town outside the city gate) of Beijing City and these two Hutong bend southward suddenly at the Wengcheng, as surrounded by moat. According to this, ZHAO believe these two Hutong are remains of the moat. It could be found that from this state of Zhao, he has realized the restriction on the historic town expansion by city wall and moat, as well as its restriction on modern street system of Beijing.

As to quantitative analysis, Zhao conducted detailed survey on the scale of streets and plots. The distance between parallel Hutong is 44 bu (about 73 meters), from which the basic units of Yuan Dynasty Beijing City can be calculated as an area of $8 \mathrm{mu}$. This sale has been confirmed in the Dongsisantiao Hutong of the modern city of Beijing. The areas of plots of important buildings in the city such as the palace, temple, etc. are modified at a certain ratio of the basic plot, which reflects the strict spatial hierarchical structure of the ancient Chinese cities.

In his study of Yuan Dynasty Beijing City, ZHAO has clearly recognized that street pattern was the most resilient element to change. Much information of development history 
Unfortunately, Zhao's death in young age made it impossible to complete his studdyang fat

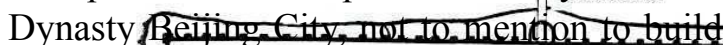
plan, XU Pingfang confirmed the accuracy of ZHAO's restoration through archaeological ans ande mande a complete methodology and terminology on fiurtherexploration on theory and mothodology. the basis of empirieat research as the western put forward the concept of the onderlapping urban mathology-schools. But these'methods of ancient and-modern-cities-and analyzes

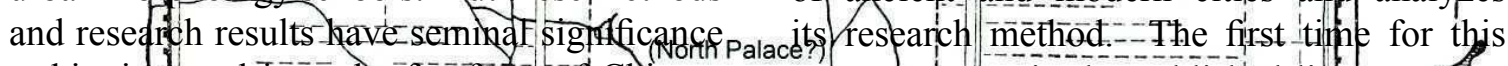
at his ting and is at the forefront of Chinese concept to use in the publishect litenature was urban study, which has a great impaction manyz cin 1972 and in 1986, Xu-Pingfang given it a

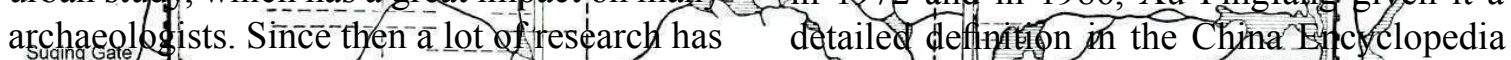

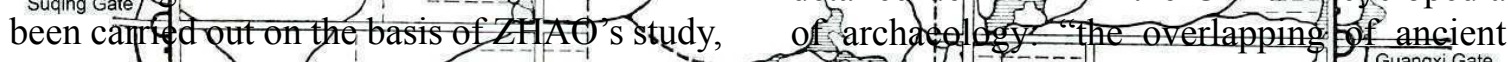
referring this experience and method, and ond wodern cities" refers-to-the city that making flither explanation and devetopment. most pats of it ane built and de ${ }^{-1}$ loped on Developnitnt of traditionat uban fierm study

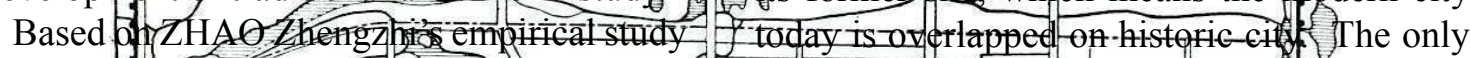
of restoration of Yan Dy nasty beijing City-2 research method for stuch cities dis to examine

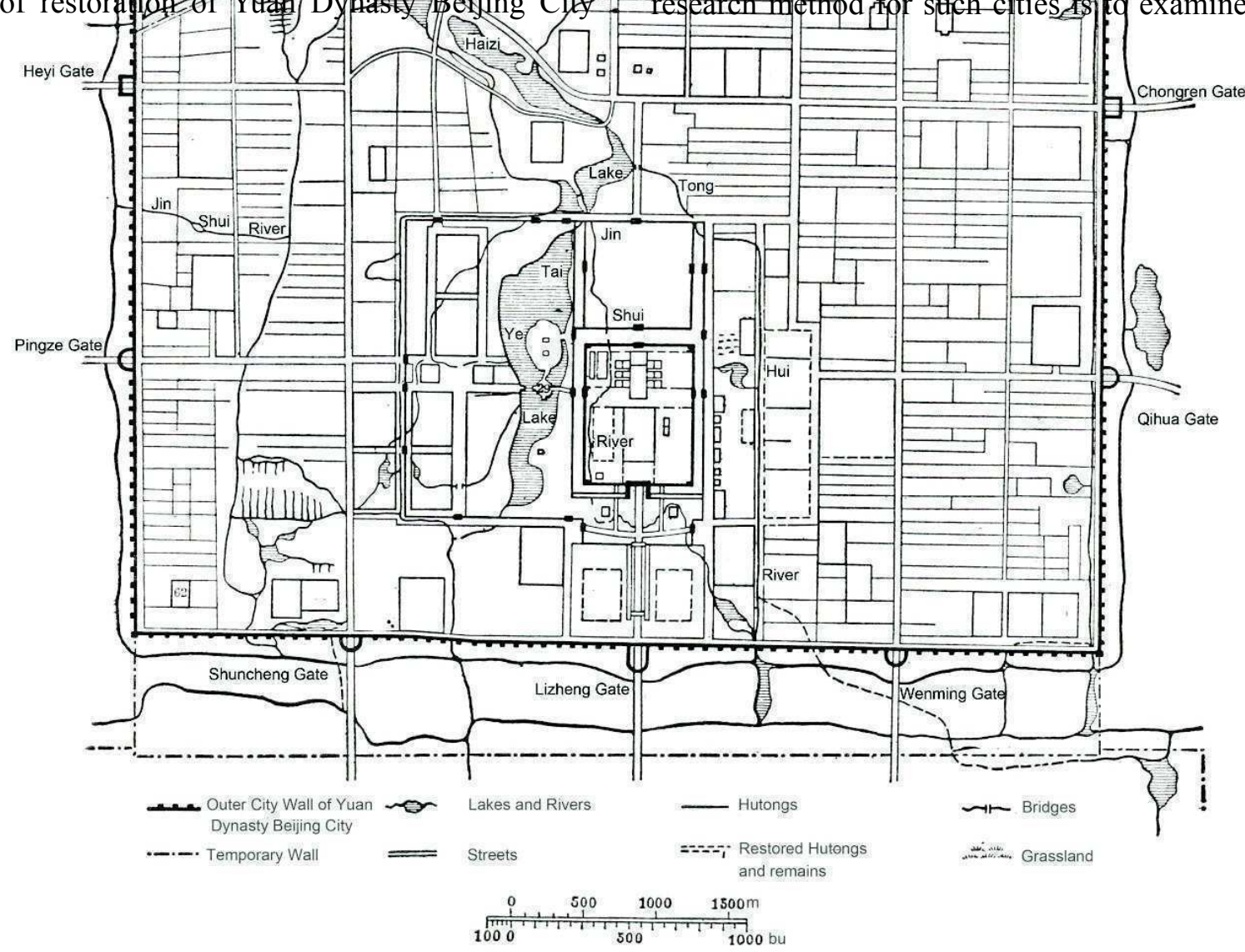

Figure 1.

Restroration Map of Yuan Dynasty Beijing City.

of the city could be reflected, and ZHAO successfully restored the historic urban plan using these clues. In his study, he combined literature information and in-depth plot level field survey investigation which linked streets and plots patterns together in analysis. At the same time, ZHAO paid attention to the limited role of restraint objects to city form, and tried to restore the develop and evolution process of the city. The scale regulation of street and plot in Yuan Dynasty Beijing City that he found by primary quantitative analysis, revealed the natural conditions, political, cultural and social characteristics behind the urban form. 
and analyze the remains of ancient cities left in modern cities. Taking Yuan Dynasty Beijing City as example, its remains could be divided into street remains, building remains and southern walls and moat remains. In these theories and methods, methods of traditional archaeological stratigraphy can be seen, but in fact, XU has already been aware of and made breakthrough of traditional archaeological methods. Much more of his emphasis is on the overall perspective of restoring ancient city form, combining methods of comprehensive literature study, mapping and prospecting. Thus the theoretical framework of urban morphology study was established.

Since then many archaeological research has used in the concept of "the overlapping of ancient and modern cities", as well as city site survey and plan restoration of historic cities carried out based on this theory. For instance, when study the historic remains in modern cities, SU Bai summarized three situations when modern cities develop on its historic site: reduction, expansion and transition, and separately discussed the spatial landscape regularity and characteristics of the overlapping of ancient and modern cities. And SU also pointed out that, it is most difficult for the restoration of original city site in the situation of transition, and he stressed the importance of identifying different times of remains and linkage of street of different times. In the restoration of Zhongzhou City, Chongqing, HANG Kan pointed out the correspondence between historical street and modern city street according to literature and historical maps study. He was also very concerned about the important role that the overlapping city theory could play in today's protection practice of historical city, and especially published articles calling for archaeologists to spread, promote and apply this theory and method to avoid wrong planning and adaptation in city regeneration. In the restoration study of the plan pattern of Zhengding ancient city published in 2017, ZHAO Xianchao still used the concepts and research methods of the overlapping of ancient and modern cities to study the changes of the ancient city plan from the Tang Dynasty to the present. As the scale of historical maps in Chinese ancient records is quite small, there is only the drawings to show relative position rather than precise geographic coordinates. In ZHAO Xianchao's study, satellite maps of 1950 s were used, combining with historical maps, literature study, historical city remains, to overcome these problems, turning the historical map into modern maps with precise coordinates.

Although China's traditional urban study has initially formed scientific research methods and system, the impact of cultural revolution against academic activities and barriers between different disciplines made it unable to play an important enough role on China's academic field and urban planning practice. Its influence was mainly limited in spread in the research field of archaeology. This has also led to the consequence that China's traditional urban morphology study was based on archaeological methods and focused on the restoration of the evolution of city plan, while paid insufficient attention to man-land relationship and urban development impetus.

\section{The spread and development of urban morphology theory in China}

Establishment of urban morphology study system

In China, the earliest use of the term chengshi xingtai (urban morphology) in urban study was the architectural architect QI Kang. In 1982, QI initially expounded the theory and method of urban morphology in his article the Form of the City (the first draft of study outline). This paper first defined the term urban form and points out its significance of study on urban form. QI takes the city as an organic whole whose form is not static but in a dynamic, evolving process. This dynamic process shows the fusion of natural and human forces and is affected by politics, social and economic factors. Qi Kang also proposed specific methods of analyzing urban morphology and its implications for urban planning practice. The research perspective QI pointed out was very advanced, which is still very basic and of great importance even in today's research.

In the field of geography study, SONG Jiatai 
and his students WU Jin, GUZhaolin, have made a great deal of instructive results as pioneers in urban morphology studies. SONG Jiatai is the first scholar who study urban form in the view of geography in China. At the beginning of 1980s he discussed the concept of city-region for the first time and expounded elements affecting and restricting the development of cities from a regional perspective. In 1990 WU Jin's doctoral dissertation, the The Morphology of Chinese Cities: Structures, Characteristics and growth is a detailed and systematic study that the concept of morphology, spatial structure, human activities and their relations were studied comprehensively. And WU explored the dynamic mechanism of morphological change from the view of morphogenesis. In the same time, WU Jin with Song Jiatai's another doctor GU Zhaolin together, focused on the studies of urban fringe area of western scholars, and then reviewed and introduced them to China at the end of 1980s. Among them J. W. R. Whitehand's study of urban fringe belt was included, and thus the concept of urban fringe area was introduced into China's geography field. After that, in 90s, they applied the theory of urban fringe to Chinese urban studies. Combined with qualitative analysis and quantitative calculation, a series of empirical studies were carried out in major Chinese cities such as Nanjing, Guangzhou and Shanghai.

Although these academic achievements in 1980s and 1990s now appears to be relatively simple and preliminary, it was groundbreaking at that time. It was these studies that introduced western urban morphology theories to China, and provided new situation for urban morphology study in geography and architecture academic field.

\section{Spread and Localization of Urban Morphology}

With the establishment of International Seminar on Urban Form (ISUF) in 1994, the theory of urban morphology has been widely disseminated. At the beginning of the $21 \mathrm{st}$ century, there have been lots of articles which introduced the theories of western urban morphology including Conzenian school and
Italian typological school, while dissemination and application of Conzenian school in a large range in China began with communication and cooperation between J.W.R.Whithand and Chinese scholars.

In 2007, Whitehand participated in the Beijing Forum 7th sub forum which discussed the inspiration of heritage to the progress of civilization. At the forum, he introduced the Conzenian school theory through Conserving Urban Landscape Heritage: a Geographical Approach and made use of the case study of Zhishanmen area in Beijing to explain the application of urban morphology in the analysis of historical urban landscape. Moreover, Whitehand, Gu Kai, Zhang Jian and other Chinese scholars did a few researches together which concerning about fringe belt and urban landscape unit in Zhishanmen area in Beijing, Pingyao in Shanxi, Hualin Temple and Tongfuxi Road area in Guangzhou. ISUF was held in Guangzhou in 2009. In this process, Whitehand, Micheal Peter Conzen and other western scholars visited China and gave lectures so that more and more Chinese researchers began to study Conzenian school and try applying its methodology to Chinese urban researches. In 2011, the Chinese version of the classic work of Conzenian School, Alnwick, Northumberland: A Study in TownPlan Analysis (translated by SONG Feng, etc.), was published. Since then, under the leadership of Song Feng, urban morphology research group in Peking University gradually formed and grew, devoted to the application and localization of Conzenian school in China. At the ISUF held in 2012, Dai Ying and Shi Yanhui reviewed the development of Conzenian school in China; Xiong Xinkai and Shi Chunhui gave a presentation on the morphological characteristics of the Land Administration Line as a special fixation line in Shenzhen Special Economic Zone; Liu Hao studied the architectural typology process of Majianglong village in southern China, it is found that under the influence of foreign culture, the village combines western building type with Chinese traditional layout. In 2013, Xiong Xiao explored the relationship between both sides of World Heritage boundary of Macau's historical center from the aspects of landscape and spatial 
hierarchy, providing an integrity perspective for World Heritage protection. Xiong Xinkai uses the method of Conzenian school to interpret the urban form of New Estates for workers in Shanghai after 1949. Wu Menghe took Tiexi District in Shenyang as an Example to explore the evolution and transformation of modern industrial landscape in China. In the same year, in the International Conference of Modern Architecture Heritage Conservation held in Xi'an, Urban Morphology Research Group of Peking University demonstrated the role and impact of Conzenian school on heritage protection through posters. At the ISUF held in 2014, Wu Menghe gave the presentation titled The Reference 8 of Chapter 2, "Alnwick, Northumberland: a Study in Town-Plan Analysis (2nd edition)" and the Research Status of Urban Morphology in China, which pointed out that M.R.G. Conzen highlighted the revolutionary planning, and paid attention to the rapid and thorough transformation with the impact of revolutionary planning in China and similar countries. Applying the theory of Conzenian school to the basic unit of the socialist spatial practice in Chinese modern city, Danwei, Dai Ying proved that the applicability of the theory and focused on the spatial characteristics as a result of influence of economic and cultural. Shi Chunhui taking an example of Nanluoguxiang, a historical district in Beijing, analyzed the important role of agent in the evolution of urban form. In 2015, basing on the plot cycle, Dai Ying and Shi Chunhui analyzed the value and perplexity of conservation and utilization of Baiwanzhuang Neighborhood, the first independently-designed neighborhood of P.R.China. Xiong Xiao studied the value and evolution process of Kuling town in Lushan, which is historical urban landscape and World Heritage, and Liu Min applied morphological methodology to settlements in West Lake, a World Heritage cultural landscape either. All of above works were completed under the guidance of Professor Song Feng, and most of them got Whitehand's support and help.

There are a number of scholars majoring in architecture focus on the researches of architectural typology. For instance, Deng Hao has completed Urban Morphology and
Typology in the United Kingdom, Basic Building Interpretation and so on, trying to break the predicament of architectural research through the interdisciplinary approach.

\section{Review of the sinicization of western morphology theory}

Looking back on the process of western morphology theory spreading and developing in China, we can recognize a clear timeline, which began with simple introduction of theory and methodology and then empirical research carried out in Chinese cities. However, in the spreading process of western morphology theory there were also errors in early geography and architecture literature. Confusion caused by cross-culture background and language barrier occurred as well. Later with more and more researchers devoted themselves to urban morphology study in China, support by western scholars, field work carried out and related reflection made, study of China's urban morphology was promoted.

In recent years, due to China's special history, differences of economic, institutional and cultural background, Chinese researchers have gradually realized the importance and necessity of the localization of western theory, and a lot of helpful exploration was made. Introducing danwei, the special socialist spatial practice and China's unique historical and cultural landscape evolution into morphological study, provide with a huge amount of detailed supplementary studies of morphological characteristics under what Conzen referred as revolutionary planning operation. Chinese scholars' exploration and efforts have never stopped for the localization development of morphological theory.

The spreading of morphology theory in China also presented obvious interdisciplinary feature, scholars of urban geography, architecture, historical geography, and even some media practitioners of heritage protection showed interest in urban morphology. Although We regret that Zhao Zhengzhi's research failed to spread widely due to the limitation of disciplinary barriers, we are more keenly aware that the general trend is breaking 
through barriers between disciplines and conducting interdisciplinary study.

\section{Impetus of morphological studies in China}

The motivation of localization of western urban morphology theory

The western urban morphology theory system has a deep historical accumulation, and with the development of history, the object of study transfer from the medieval historical town gradually to the contemporary urban form. As a result of special historical development, China's urban development process lags behind that of western developed countries, and the urban issues in development show a certain lag too. Accordingly, the study of urban morphology present lack in theoretical depth and the public policy to deal with these problems also vacant in some area.

Nevertheless, the problems in urban development arisen in China and the Western countries still show considerable similarity, which makes it possible and necessary for China to learn and draw lessons from western theories and experiences. Chinese cities have experienced a period of almost 40 years' rapid development after reform, abundant cases for contemporary urban morphology could be found. Although Chinese cities are different from the western cities in term of historical development, there are also develop stages and periodicities, whose effects are clearly reflected in the material form of city (TIAN Yinsheng, 2010). The concept of plan unit, fringe belt in Conzen school and the special concern on special Building in Italian architectural typology provide new solutions to current problems of Chinese cities. Especially in the conservation and management of historical cities and historical streets, the relevant western urban morphology theories show strong applicability.

However, we can not ignore the characteristics of Chinese cities while drawing lessons from western urban morphology theories. Facing diverse historical context and modern institution, although the urban problems show some similarities in spatial level, behind the phenomenon is the difference of economic and cultural background. This reminds us to pay more attention to the adaptability and localization of the western urban morphology theory when we apply that to explore the solution to Chinese urban issues. Therefore, it is particularly important to trace the research context of Chinese indigenous urban morphology studies. Based on the study of indigenous urban morphology theory, applying the western urban morphology theory adaptively as well as localizing it to makes it more suitable for Chinese urban morphology study.

\section{Support from western scholars and ISUF held in China}

In 1970s and 1980s, the development of urban morphology in China set off a climax. The study of urban morphology has become more and more eye-catching, and the meaning and understanding of urban morphology has been initially unified (Li Yang, 2006). With the rapid development of Chinese cities in the new century, the study of urban morphology has entered a period of rapid development too. During this period, the introduction of western urban morphology to China played a key role, especially thanks to the enthusiastic help and promotion from a number of western scholars. The Urban Morphology Research Group (UMRG) is the earliest urban form research organization in the UK, initially established by Jeremy Whitehand, Terry Slater and a group of other young scholar of human geography in the University of Birmingham in 1974. UMRG has become one of the most active academic organizations in the field of urban morphology and in decades, its influence has gradually expanded from Europe to the whole world (Zhang Jian, 2012). In the background of rapid development of cross cultural urban form study in recent years, UMRG has supported to establish China's urban morphology research group in South China University of Technology and the Peking University, carrying out morphological study with the theory of Conzenian school.

As the main member of UMRG and professor 
of geography at the University of Birmingham, Jeremy Whitehand has made a great contribution to the spread and development of Conzenian school urban morphology theory in China. Jeremy has been to China to give lectures for several times and under his guidance, Chinese scholars have carried out morphological studies in Beijing Zhishanmen historical area (2007), Pingyao City (2010), Kuling town Mount Lushan (2013), and Shangqiu (2016). A number of Chinese scholars also visited UMRG at the University of Birmingham to further understand the Conzenian urban morphology theory. Micheal Conzen and other scholars made great efforts to the spreading of urban morphology theory in China as well.

In 1994 International Seminar on Urban Form was established by UMRG and Italian architectural typology scholars, and after over twenty years of development, ISUF has become the most important platform for international exchanges of studies in the field of urban form. In the dissemination and development of the West urban morphology theory in China, there are two ISUF held in China: the 16th ISUF was held South China University of Technology in Guangzhou, 2009, and the 23rd ISUF held in Nanjing University in Nanjing, 2016.

The two ISUFs held in China not only showed the spreading of urban morphology in China, but also brought up a number of topics related to China, reflection on China's study of urban form, which aroused extensive discussion. In the 2016 ISUF in Nanjing, there were nearly $60 \%$ among the 140 papers were from Chinese scholars, including Peking University, Southeast University and other major scientific research institutions and universities. The urbanization issues, plot and building type of historic city, study method for urban morphology were discussed.

\section{Commemoration and prospection}

Contributions can not be ignored

The development of urban morphology in China depends on the foundation of Chinese and Western scholars' researches, research on the town plan of Yuan Dynasty Beijing City did by Zhao Zhengzhi, research on the town plan of Alnwick did by M.R.G.Conzen are all the monumental groundbreaking works, which never too much to be reviewed. Coincidentally, Zhao Zhengzhi and M. R. G. Conzen both born in 1907 and their researches were both completed around 1960, but due to the difference in academic environment and the premature death of Zhao Zhengzhi, two equally important studies received totally different attention.

2017 is the 110th anniversary of Zhao Zhengzhi and Conzen's birth. Reviewing classics of urban morphology is the best way to express the commemoration and memorial.

Review and prospection

This paper summarizes the morphological researches in the field of archeology, geography and architecture, and through the review of predecessors' contributions and the integration of existing researches, clarifies the origin and development of urban morphology in China, so as to arouse the regard and reflection on academic tradition.

The dissemination of morphological theory in China is the process of combining theory with practice and localizing methodology in China. The difference of the urban morphological studies between western countries and China mainly comes from two aspects: the revolutionary transformation caused by different land system and planning means, and the spatial layout with politics core rather than economic core caused by traditional culture. It is the direction for Chinese researchers to establish theory and methodology system which is more suitable for Chinese practice based on the works of predecessors.

\section{Reference}

Conzen, M. R. G. (2011) Chengzhen Pingmian Geju Fenxi:Nuosenbolanjun Annike Anli Yanjiu (Alnwick, Northumberland: A Study in Town-Plan Analysis), translated by Song,F., Zhongguo Jinazhu Gongye Chubanshe(China Architecture \& Building 
Press, Beijing.)

Dai, Y., Shi, C., Song, F. (2015) 'Morphology cognizes the heritage of urban and rural spatial practice of socialist China, exemplified by Baiwanzhuang neighborhood in Beijing', Chengshi Fazhan Yanjiu (Urban Development Studies) 23, 49-55.

Dai, Y., Shi, Y., Song, F. (2012) 'Research progress of Conzenian school in Peking University', ISUF 2012.

Dai, Y., Song, F. (2014) 'Danwei as an agent of change in modern socialist China, exemplified by Hongta Tobacco (group) Co., LTD', unpublished thesis.

Gu, C. L. and Xiong, J. B. (1989) 'Jianshu chengshi bianyuanqu yanjiu'('On urban fringe studies'), Dili Yanjiu(Geographical Research)03, 8(3): 95-101.

Gu, C., C, T., D, J. H. and Y, W. (1993) 'Zhongguo dachengshi bianyuanqu texing yanjiu' ('The study of the urban fringes in Chinese megalopolises'), Dili xuebao( Acta Geographica Sinica) 48(4), 317-28.

Liu, H., Song, F., Deng, H., Xiong, X., Shi, C., Dai, Y., (2012) 'Majianglong Villages: typological process analysis', unpublished thesis.

Nanjing Daxue Renwen Dili Yanjiu Zhongxin(Research Center of Human Geography). (21 Jan 2011), 'Song Jiatai Jiaoshou'('Professor Song Jiatai'),available at: http://hugeo.nju.edu.cn/ geoer/779(accessed 20 Sep 2012).

Qi, K. (1982) 'Chengshi de xingtai(yanjiu tigang chugao'('Urban Morphology (The initial draft of the Outline of the Study) '), Dongnan Daxue Xuebao(Journal of Southeast University)3, 14-27.

Qi, K. (2011) 'Chengshi de xingtai' ('Urban Morphology'), Xiandai Chengshi Yanjiu(Modern Urban Research)5, 92-6.

Shi, Y., Dai, Y., Xie, N. (2014) 'Culture landscape: from academic origin to protection of world heritage', Zhongguo Yuanlin (Chinese Landscape Architecture) 30, 78-81.

Song, F. (2012) 'Zaidu Zhaozhengzhi Xiansheng <Yuandadu Pingmian Fuyuan de Yanjiu $>$ ' ('Reread the work of Zhao Zhengzhi: Research on the town plan restoration of Yuan Dynasty Beijing City'),
In Zhongguo kaogu xuehui(Archaeological Society of China) and Shenyangshi Wenwu Kaogu Yanjiusuo(Institute of cultural relics and archaeology of Shenyang)(eds), Qingzhu Subai Xiansheng Jiushi Huadan Wenji(Corpus in celebration of Subai's Nineties birthday) ( Science Press, Beijing) 355-62.

Song, F. (2012) Re-exploration on the research method of 'superposition clusters', unpublished thesis.

Song, J. (1995) 'Guanyu chengshi xingtai'('About urban morphology of China'), In Song J. (eds) 'Songshi Wengao Chuji'('Mr. Song's Manuscripts Early Set'), unpublished book,287-9.

Whitehand, J. W. R. (2007) 'Urban conservation in China: Historical development, current practice and morphological approach', Town Planning Review78,643-70.

Whitehand, J. W. R. (2012) ' Issues in urban morphology', Urban Morphology 16(1), 5565.

Whitehand, J. W. R. and Gu, K. (2006) 'Research on Chinese urban form: retrospect and prospect', Progress in Human Geography, 30(3), 337-355.

Whitehand, J. W. R. and Gu, K. (2007) 'Conserving Urban Landscape Heritage:a Geographical Approach', in Beijing forum academic committee of Peking University. (Ed.), The Harmony of Civilization and Prosperity for All(diverse development model of human civilization ): Selected Papers of Beijing Forum(2007), (Peking University press, Beijing) 284-293.

Whitehand, J. W. R., Gu, K. and Whitehand, S. (2011) 'Fringe belt and socioeconomic change in China', Environment and Planning: Planning and Design, 38(1) 41-60.

Whitehand, J. W. R., Gu, K., Whitehand, S. and Zhang, J. (2011) 'Urban morphology and conservation in China', Cites, 28(2) 171-185.

Wu, J. (1990) 'Chengshi bianyuanqu kongjian jiegou yanhua de jizhi fenxi'('Analysis of the evolution mechanism of the spatial structure of the urban fringe'), Chengshi Guihua(City Planning Review) 02,38-42.

Wu, J. (1990) 'Zhongguo chengshi bianyuanqu kongjian jiegou tezheng jiqi fazhan: yi 
Nanjing deng chengshi weili' ('The spatial structure and development of Chinese urban fringe: taking Nanjing as an example'), Dili Xuebao(Acta Geographica Sinica) 45(4), 399-411.

Wu, J. (1990) Urban morphology of China: Structure, characteristics, and its evolution. Nanjing: Phoenix Science Press.346-53.

Wu, M., Shi, C, Song, F. (2014) 'The reference 8 of Chapter 2, "Alnwick, Northumberland: a Study in Town-Plan Analysis (2nd edition)" and the research status of urban morphology in China', ISUF 2014.

Wu, M., Song, F. (2013) 'The changing face: transformation of modern industrial landscape in China', ISUF 2013.

Xiong, X., Dai, Y., Song, F. Lu, Y., Zhu, D. (2015) 'Morphology-based identification to OUV of HUL and its geographical process: a case study of Kuling Town, Lushan', Renwen Dili (Human Geography) 32(03), 36-43

Xiong, X., Shi, C., Song, F., Zhao, X. (2012) 'A special fixation line in Shenzhen, China', unpublished thesis.

Xiong, X., Song, F. (2013) 'Reading heritage from the perspective of city', ISUF 2013.

$\mathrm{Xu}$, P. (2001) 'Lun lishi wenhua mingcheng Beijing de gudai chengshi guihua jiqi baohu' ('On ancient urban planning and protect of historic city Beijing'). Wenwu (Cultural Relics) 1, 64-73.

Zhao, Z. (1979) 'Yuandadu pingmian guihua fuyuan de yanjiu'('Research on the town plan restoration of Yuan Dynasty Beijing City'), Kejishi Wenji (Corpus of History of Science and Technology) 2, 14-27. 